\title{
Las mujeres van a la guerra
}

\author{
Editado por/Edited by: David Vásquez L. \\ Recibido/Received: 26/01/2014. Aceptado/Accepted: 15/05/2014 \\ Publicado en línea/Published on Web: 10/09/2014
}

\section{Cristian Mejía}

Universidad San Francisco de Quito, Colegio de Ciencias Sociales y Humanidades COCISOH

Diego de Robles y Vía Interoceánica, Quito, Ecuador

Correo electrónico: cristian.mejia.obando@estud.usfq.edu.ec

\section{David Revelo}

Universidad San Francisco de Quito, Colegio de Ciencias Sociales y Humanidades COCISOH

Diego de Robles y Vía Interoceánica, Quito, Ecuador

Correo electrónico: fabian.revelo@estud.usfq.edu.ec

\section{Valeska Yánez}

Universidad San Francisco de Quito, Colegio de Ciencias Sociales y Humanidades COCISOH

Diego de Robles y Vía Interoceánica, Quito, Ecuador

Correo electrónico: valeska.yanez@estud.usfq.edu.ec

\section{Resumen}

\begin{abstract}
Este artículo explora el debate académico que existe en torno al rol que cumple el género en los conflictos armados. Históricamente se ha creído que las mujeres tienen un papel secundario en la práctica de la violencia en conflictos internos, centrándolas como víctimas y no como victimarias. Se ha pretendido sustentar estas afirmaciones con estudios psicológicos y prejuicios de género. Por este motivo, el enfoque que este artículo propone hace referencia también a estudios psicológicos pero además toma estudios acerca de conflictos armados internos en los que las mujeres han tenido protagonismo. Pretende sustentar que las mujeres son igual de violentas que los hombres. Este argumento se apoya en la ya demostrada participación activa de las mujeres como efectivos militares en enfrentamientos bélicos acaecidos en conflictos post Guerra Fría y en los diferentes tipos de violencia que ellas perpetran. Como estudio de caso se tomará al conflicto en Siria.
\end{abstract}

Palabras clave: guerra, género, Siria, violencia, mujeres.

\section{Abstract}

This article explores the existing academic debate around gender and its role in conflict. It has been historically believed that women have had a secondary role in the exercise of violence, portraying them as victims rather than perpetrators. There have been attempts to support these claims with psychological studies and gender-biased preconceptions. For this reason, the approach sustained in this article also refers to psychological studies but focusing on studies of internal armed conflicts in which women have had prominence. The article aims to support the thesis that women are as violent as men. This argument is based on the proven and active participation of women as soldiers in armed conflicts performed throughout the post-Cold War conflicts and the different types of violence they perpetrated. The conflict in Syria will be the analyzed as a case study to prove this thesis.

Palabras clave: war, gender, Syria, violence, women.

Al hablar de guerra seguramente lo primero que se viene a la cabeza son pistolas, ametralladoras, bombas, granadas, etc. Pero con seguridad también, aparece la idea de que las personas que manipulan esas armas son en su totalidad hombres. El balance de género y más que nada la inclusión de las mujeres en la guerra no ha sido un tema de relevancia: en Estados Unidos en 1973 tan solo el 1.6\% de la armada eran mujeres (Becraft, 2005, pág. 1). Las mujeres han sido segregadas de la vida militar por varios aspectos, en los que se pueden resaltar dos grupos biológicos y psicológicos, como los más importantes. Antes de empezar a tratarlos es necesario definir qué es la guerra pues el artículo se referirá a conflicto. Guerra es un conflicto militar entre dos o más partes, por lo menos deja 1000 caídos en combate en un período de 12 meses, generalmente se da entre el Estado y un grupo de la sociedad civil (Sarkees \& Wayman, 2010). Por otra parte también es necesario dejar en claro el concepto de género. Según la Organización Mundial de la Salud (OMS, 2014) el género "se refiere a los conceptos sociales de las funciones, comportamientos, actividades y atributos que cada sociedad considera apropiados para los hombres y las mujeres" (OMS, 2014). Es importante tomar en cuenta estos conceptos a la hora de analizar el equilibro de género en la guerra.

Las mujeres no siempre estuvieron vinculadas con la milicia, de hecho siempre fueron un tema aparte en la sociedad. En 1793 las mujeres fueron excluidas de todos los derechos polí- 
ticos estrenados, se ordenó que se disuelvan todos los clubes femeninos, se prohibió una reunión de más de 5 mujeres en la calle y la desobediencia se castigó con la guillotina. Esto sucedió en el desarrollo de la primera ola del feminismo en Europa y Estados Unidos (Varela, 2005, pág. 41). El ser mujer era ser diferente, romper el orden establecido. Simone de Beauvoir, en El Segundo Sexo, "expone la teoría de que la mujer siempre ha sido considerada la otra cosa con relación al hombre, sin que ello suponga reciprocidad" (Varela, 2005, pág. 84). Beauvoir afirma que los hombres son los detentadores del poder y creadores de la cultura [...] en un mundo dominado por hombres las mujeres no tienen su oportunidad" (Varela, 2005, pág. 85).

La conclusión a la que llega es que no hay nada biológico ni natural que explique la diferenciación de hombres a mujeres. Lo que ha ocurrido es que la cultura ha dado más valor a quien arriesga la vida, que es lo que los hombres hacen en las guerras, que a quien se las da, que es lo que hacen las mujeres con su poder de concebir (Varela, 2005, pág. 87). La filósofa insiste en separar el sexo, lo biológico, de lo que la cultura dicta para hacer en caso de ser hombre o mujer, que ahora se conoce como género y es tan sólo una construcción social que produce segregación e inequidad.

Cuando se desarrollaron las Guerras Mundiales y algunos de los conflictos armados más relevantes en el mundo, los estereotipos eran demasiado fuertes, es decir, los hombres jugaban un papel de héroes, pues sacrificaban su tiempo, su familia, sus miedos y su cuerpo para ir a matar o morir por una causa. Las mujeres, al contrario, eran consideradas pacifistas ingenuas ante el suceso de una guerra y debían ser protegidas por los guerreros (Sjoberg, 2010). Es importante también mencionar que las mujeres cumplían un papel importante en la industria nacional cuando los hombres acudían a una Guerra interestatal.

Si bien es cierto que en guerras de colonización sucedidas hace cientos de años ya había participación femenina, ésta no era de manera formal. Como explica Sjoberg, las mujeres que luchaban tenían que adoptar un papel masculino para ser aceptadas en los servicios militares, cambiaban su vestimenta, aprendían estándares físicos de los hombres y a comportarse de manera varonil (Sjoberg, 2010). Con el pasar del tiempo la milicia se flexibilizó y las mujeres empezaron a tener roles formales, mas estos roles se limitaban a la enfermería y la cocina. No fue hasta 1939,

...en la Segunda Guerra Mundial cuando se puso de relieve su papel, fundamentalmente como reservistas o como unidades de apoyo [...] en las fuerzas alemanas y británicas; en el caso de la Unión Soviética, su participación directa en la lucha como miembros de todos los servicios y unidades ascendió al 8\% del total de las fuerzas armadas" (Lindsey, 2000).

Luego de este incidente las mujeres han participado de manera activa, mas su participación y reclutamiento son reducidos y hay un dilema en cuanto a la aceptación de mujeres dentro del conflicto armado. No obstante, la teoría que aquí pretendemos esbozar se basa en el hecho de que las mujeres, tal y como sus contra partes masculinas, no solo pueden ser objetos de violencia, al contrario, pueden ser sujetos que ejercen activamente métodos violentos para alcanzar sus objetivos y, por tanto, son eficientes como integrantes de grupos armados.

Existe un debate académico cuando se presentan las razones por las que las mujeres no tienen presencia significativa en los conflictos armados. Dentro del ámbito de las Relaciones Internacionales, campo de nuestra competencia, se discuten especialmente dos corrientes: el feminismo de la igualdad y el feminismo de la diferencia. En primer lugar está el feminismo de la diferencia, que toma el término 'diferencia' y le da otro sentido, reivindica el concepto y se basa justamente en la diferencia de las mujeres para establecer una identidad propia. Una de sus ideas claves es señalar que 'diferencia' no significa desigualdad. Esta corriente promueve la igualdad entre hombres y mujeres pero no la igualdad con los hombres, ya que esto implicaría aceptar el modelo masculino (Varela, 2005, pág. 120). Por otra parte tenemos al feminismo liberal que busca que las mujeres ocupen espacios que históricamente han sido ocupados por los hombres, supone que hombres y mujeres son iguales en habilidades y que segregarlas es un acto de discriminación (Goldstein, 2001, pág. 39).

Al momento de analizar la guerra, estas teorías nos dan una perspectiva de cómo ve el mundo la participación de las mujeres. En la otra cara de la moneda pero dentro del mismo ámbito de las Relaciones Internacionales, el argumento de muchos teóricos es que las mujeres, al contar con instintos maternales, potencial de protección y una bondad innata, se opondrían a la guerra (Goldstein, 2001, pág. 34). Esto, al sacarlo del nivel doméstico, y llevarlo a nivel estatal, provocaría un cambio en las instituciones antiguamente creadas por hombres ya que las mujeres cumplirían un papel de peacemakers y en un nivel global se podría decir que un mundo gobernado por mujeres sería más pacífico.

La masculinidad es uno de los requisitos para ser un buen guerrero, pues la creencia popular apunta a que los hombres están naturalmente preparados para ir a la guerra desde su condición genética. Ellos cuentan con mayor fortaleza y resistencia, menos fatiga, mientras "las mujeres sufren desórdenes psicológicos post-traumáticos con mayor frecuencia que los hombres y, en general, acusan secuelas negativas mayores tras ser sometidas a algún tipo de agresión física" (Candil, 2007). Por último se supone que al insertar mujeres al conflicto, los hombres presentarían altos niveles de desconfianza hacia las mujeres por no tener una serie de cualidades óptimas para estar en la guerra y aparte por su gran emotividad, esto influiría en un mal desempeño en el conflicto.

Pero en realidad ¿las mujeres no son aptas para ir a la guerra? ¿Existen e influyen estas diferencias biológicas en un conflicto armado? ¿Un mundo gobernado por mujeres sería más pacífico?

\section{Diferentes perspectivas}

Los anteriores argumentos se fundamentan en las diferencias biológicas y psicológicas que supuestamente existen entre hombres y mujeres; no obstante, entra también al debate una contraparte que analiza el género, la cultura y cómo los roles sociales son muchas veces el limitante más grande que tienen las mujeres. En realidad, las mujeres y los hombres no presentan diferencias mayores o que influyan decisivamente en el conflicto. Existen diferentes perspectivas con las que se puede argumentar esto. 


\section{Perspectiva biológica}

$\mathrm{Si}$ analizamos la parte biológica, podemos empezar por la estructura genética de las mujeres y los hombres. Las mujeres presentan una combinación XX y los hombres XY (Derisas, 2012). Hombres con una combinación XYY no son más violentos a pesar de tener un extra de Y. Los genes por sí solos no son un indicador válido sobre el desempeño de los hombres y mujeres en la guerra. Los genes son parte de una estructura que lo que hace es pasar de generación en generación la información necesaria para que el cuerpo fabrique las proteínas necesarias para la vida. Estas proteínas son las que dan como resultado final la producción de las hormonas que a la larga definen los fenotipos de los sujetos (Goldstein, 2001). Si hablamos de hormonas, la testosterona, estrógenos y progesterona, están todos presentes en el cuerpo humano. El hecho es que unos se manifiestan en un tiempo y circunstancia determinada, lo cual no quiere decir que se encuentren en potencia presentes sin existir diferencia. La testosterona y su producción tienen cierto tipo de relación con el comportamiento de los sujetos pero se encuentra tanto en hombres como en mujeres y por tanto impacta en ambos (Norman \& Lidwack, 1987).

\section{Desarrollo Cognitivo}

El desarrollo cognitivo de los hombres, es evidentemente diferente al de las mujeres. Por ejemplo. Los hombres son mejores en habilidades de ubicación espacial y pensamiento numérico. En tanto, las mujeres tienden a ser mejores en desarrollo verbal, y pensamiento de precisión (Goldstein, 2001).

\section{Perspectiva psicológica}

Las evidencias muestran que las mujeres, en ambientes dominados por hombres, tienden a aceptar el status quo e inician un proceso de adaptación mediante la "cooptación" (Bystydzienski, 1993), que consiste en llenar las vacantes del grupo e ir escalando posiciones. Además, imitan el comportamiento violento de los hombres pues deben ser un espejo de sus compañeros. Incluso las mujeres pueden ser tan violentas que atacan a hombres que siendo víctimas refuerzan el sentimiento de pertenencia e igualdad de las mujeres en su grupo (Carpenter, 2003). En movimientos anticolonialistas o revolucionarios, que usualmente luchan en contra de algún régimen opresor, los roles de género se pueden difuminar y confundir, pues la lógica de la necesidad llega a superar a la lógica de lo convencional (Alison, 2004). Por tanto si una comunidad está bajo ataque, los roles que se definían en tiempos de paz como lo que debe ser se cambian, por necesidad imperante, a lo que tiene que ser; es decir, que los papeles entre hombres y mujeres pueden variar de manera radical en un contexto de estrés y necesidad (lo que tiene que ser), de lo que era en tiempos de paz (lo que debe ser). Y es en estos momentos que la situación de lo femenino cambia y se transforma en una mujer siendo capaz de defenderse a sí misma y a sus semejantes sin necesidad de un hombre, y pudiendo ser igual o más violenta que sus compañeros masculinos.

\section{Roles de Género}

En general, como ya se dijo, la violencia perpetrada en contextos de conflicto armado, ha sido tomada como mérito masculino, y aun desde las perspectivas más feministas, la mujer no ha sido vista como victimaria, es decir, solo ha sido vista como víctima. Psicológicamente los sujetos femeninos que se encuentran inmersos en grupos radicales y violentos son toma- das como miembros que han fracasado o son deficientes en su proceso de socialización, por lo cual encuentran refugio en este tipo de comunidades. Además se afirma que son sujetos que están más alejados de la realidad que sus contra partes masculinas (Zwerman, 1997, pág. 136).

Esto pone a las mujeres en un papel de "enfermas mentales" que ejercen violencia solamente por sus disfunciones psicológicas y que no llevan detrás de sus acciones elementos políticos o ideológicos como lo pueden hacer los hombres (Blake, 2005, pág. 43). Este aspecto del comportamiento femenino es ignorado porque amenaza la visión preestablecida de pasividad y feminidad que se tiene del género femenino. La participación de las mujeres en la violencia subversiva armada no solo rompe con la ley jurídica convencional, al contrario, rompe con la ley social de "lo que debe ser".

La parecencia de la mujer en este tipo de actividades no se da solamente por el hecho de que puede ser; se da por el hecho de que algunas de esas mujeres quieren desafiar el convencionalismo del género y el papel preponderante que se le da en la sociedad (Zwerman, 1997, pág. 131). Por tanto, existe un rol binario de lo femenino en el ejercicio de la violencia pues la mujer no solo lucha contra el enemigo, al contario lucha también contra la inseguridad social en la que vive (Alison, 2004). En este aspecto binario se figura la realidad de que las mujeres pueden estar ahí para demostrar el simple hecho de que ellas también pueden ser útiles en estos contextos (Bloom, 2011) y de que no hay nada que las diferencie o las haga menos capaces que los hombres.

Es por eso que las mujeres en grupos subversivos o en grupos terroristas intentan conseguir de una u otra manera superar a sus contrapartes masculinas asesinando a más personas, o en otras situaciones son capaces de violar a personas de su mismo sexo (Brownmiller, 2013). Las mujeres no pueden escapar de la psicología que se manifiesta en el grupo, pues en situaciones de violencia armada los grupos necesitan cohesión dentro de sí. La violación en masa, aun cuando las violadoras sean mujeres, responde a esa necesidad grupal (Cohen, 2013) que impera sobre la identidad de género. En estas situaciones las víctimas han perdido toda capacidad de representar humanidad ante el victimario, es decir, han sido deshumanizadas (Zalles, 2004).

En conclusión, los marcos teóricos que se han construido alrededor del estudio de los conflictos armados están cargados de inobservancias sesgadas por prejuicios de género (Carpenter, 2003). Las mujeres pueden ejercer violencia en contextos de conflicto armado tal como lo pueden hacer los hombres, sin diferenciarse en demasiados aspectos del ejercicio de dicha violencia. Los agentes femeninos tampoco pueden escapar de la lógica y las necesidades que se manifiestan al interior de un grupo armado. Por tanto, las mujeres también van a la guerra.

\section{Estudio de caso: Siria}

Siria, ha vivido un conflicto armado interno que tuvo como origen graves disturbios internos y una escalada de violencia de magnitud humanitaria. Las revueltas populares del 28 de Enero de 2011 desataron una ola violenta que aún no termina. De acuerdo al Alto Comisionado de las Naciones Unidas para los Refugiados (ACNUR), existen en Siria alrededor de 2 millones de refugiados y más de 500.000 son mujeres entre 
18 y 30 años. Las partes en conflicto son las fuerzas militares del gobierno del Presidente Bashar Al-Assad y las fuerzas de oposición nombrados normalmente como la "Coalición". El grupo de oposición es diverso: existen desde milicias del pueblo Kurdo hasta grupos islámicos extremistas apoyados por Al-Qaeda, como es el caso del frente Al-Nusra (Diehl, 2012). Mientras tanto, la milicia de Bashar Al-Assad está formada por varios frentes, algunos conformados por militares y otros por civiles. Las mujeres, reclutadas por ambos bandos en conflicto, han sido entrenadas con ferocidad.

Los roles de género apuntan a que las mujeres enlistadas en conflictos armados son meramente disidentes sociales. Quienes han fracasado y son seres violentos son quienes encuentran refugio en comunidades igualmente "violentas". Ciertamente, esta teoría es incorrecta si lo contrastamos con el caso de Siria. Syrian Report afirma que un 52\% de las mujeres enlistadas en los diferentes bandos en conflicto no solo son madres o abuelas. Son profesionales (abogadas, economistas, catedráticas, etc.) quienes han decidido salir a luchar debido a que no existía otra forma de contrarrestar la violencia en sus comunidades (Syrian Report 2013).

De acuerdo con Joshua Landis, Director del Centro de Estudios de Medio Oriente de la Universidad de Oklahoma, las mujeres soldado deberían ser escasas en Siria debido a que las partes en conflicto están repletas de fundamentalistas religiosos (Cheikhomar, 2013). Pero, para sorpresa de muchos, cada día más mujeres se unen a ambos bandos del conflicto y no como enfermeras, cocineras o activistas. Lo hacen de forma directa, tomando un rifle y asesinando antes de ser asesinadas (Syrian Report, 2013). El primer grupo de mujeres fue reportado a inicios del 2013 en la ciudad Siria de Homs. Hoy en día se registran al menos 6 frentes armados femeninos, de ambos bandos del conflicto.

Dentro del marco de la psicología, las mujeres en Siria han adoptado el status quo de cierta manera, no por el desarrollo cognitivo de un grupo frente a otro. Esto se debe a que el entrenamiento que reciben es pobre y similar al de las tropas masculinas (Esman, 2014). Esto conlleva a que el grupo dentro del cual se desenvuelven hombres y mujeres se convierta en homogéneo (Esman 2014). Convierte a las mujeres en rivales verdaderos frente a los hombres debido a que esta supremacía masculina desaparece. Poco se sabe de la forma de entrenamiento sádico al cual someten a los soldados en Siria durante tiempos difíciles. Durante la guerra civil en Siria, en la administración de Hafez Al-Assad, se le daba importancia al entrenamiento femenino sobre el masculino, eliminando a las mujeres de sus deseos débiles, como el sentimiento maternal, por medio de prácticas dolorosas como golpizas, trabajos forzados e incluso consumo de drogas (Elgot 2013).

Uno de los grupos más sanguinarios de guerra bajo el mando del gobierno es la Fuerza de Defensa Nacional, que empezó a adiestrar mujeres en frentes de defensa en las principales ciudades sirias. Dentro de este grupo se encuentran las Leonas de Siria, una facción formada en su totalidad por mujeres, quienes gritan a viva voz: “Alá, Siria y Bashar". Han sido desplazadas en mayor medida en la ciudad de Homs y han sido entrenadas para utilizar kalashnikovs, granadas y equipo pesado en general (Esman, 2014).

Por otra lado, en la ciudad de Aleppo, las unidades de combate femeninas de la "Coalición" custodian cuidadosamente las calles. Se hacen llamar a ellas mismas Al Mouminin Aisha, en honor a la esposa del profeta Mohammed. Muchas de estas mujeres son orgullosamente musulmanas sunís y dicen que anhelan el establecimiento de un Estado Islámico en Siria. La líder de este movimiento de mujeres en Aleppo es Um Mohammed, madre de cuatro hijos y esposa de un soldado del Frente de Liberación Sirio (Elgot, 2013).

Ella no es el único testimonio de mujeres que han decidido tomar la protección de sus vecindarios o villas por mano propia. Esta facción armada en la ciudad de Aleppo, conformada por 130 mujeres aproximadamente, aseguró haber asesinado al menos 8 soldados del gobierno cada una (German, 2013). Una de las líderes de esta facción afirma que muchas mujeres ofrecen su ayuda en hospitales u orfanatos pero que ellas han decidido salir al campo y jugarse la vida por un cambio de raíz en el gobierno siria (Elgot, 2013). Las mujeres, no están jugando un rol pasivo en Siria, a pesar de las barreras culturales; están demostrando que pueden ser tan fuertes y agresivas como los hombres.

El conflicto en Siria sigue latente. Ambos bandos continúan siendo fuertes y el pueblo lucha por su supervivencia. Los frentes armados femeninos son nuevos, empezaron a formarse en 2012 y existe información limitada sobre los avances de varios de ellos. Lo único que es seguro es que las mujeres han demostrado romper cualquier barrera de género e, incluso en una región tan compleja como Medio Oriente, han tomado su seguridad en sus manos.

El caso en Siria demuestra que las barreras de género no han sido un impedimento para el enrolamiento de la mujer en las guerras. En lugar de eso han impulsado la creación de grupos armados únicamente femeninos, quienes luchan al igual que los hombres por salvar sus vidas y la de sus seres queridos. Debido al entrenamiento que reciben, las mujeres se han desprendido de la posición de inferioridad frente a la sociedad patriarcal y pueden ser vistas como iguales frente a sus contrapartes masculinas. Siria, es un caso muy especial, debido a que no es la primera vez que las mujeres han luchado en grupos armados. Es importante recalcar que la información sobre mujeres soldado en Siria es escasa pues no existen registros de los grupos femeninos constituidos por Hafez Al-Assad durante la guerra civil en los ochentas y muy poca ahora sobre las milicias femeninas.

\section{Referencias}

Alison, M. (2004). Women as agents of political violence: Gendering Security. Security Dialogue, 447-463.

Austin, H., \& Cheikhomar, A. (23 de Junio de 2013). They can't succeed without us': Women take front-line role in Syria conflict. Recuperado el 2014, de NBC News: http://worldnews.nbcnews. com/_news/2013/06/23/19076204-they-cant-succeed-withoutus-women-take-front-line-role-in-syria-conflict?lite

Baron, R., \& Richardson, D. (1994). Human Agression. New York: Plenum Press.

Becraft, C. (19 de Enero de 2005). Facts About Women in the Military, 1980-1990. Recuperado el 18 de Febrero de 2014, de Feminism and Women's Studies: http://feminism.eserver.org/workplace/professions/women-in-the-military.txt 
Bloom, M. (2011). Bombshell: The Many Faces of Women Terrorists. Toronto: Penguin.

Brownmiller, S. (1975). Against Our Will: Men, Women and Rape. New York: Simon and Schuster.

Bystydzienski, J. (1993). Women in Groups and Organizations: Implications for the Use of Force. En R. H. Howes, \& M. R. Stevenson, Women and the use of military force. Boulder: Lynne Rienner.

Candil, A. (30 de Noviembre de 2007). ¿Deben las mujeres ir a la guerra? Recuperado el 10 de Marzo de 2014, de Grupo de Estudios Estratégicos: http://www.gees.org/articulos/deben_ir_las mujeres_a_la_guerra_4776

Caprioly, M., \& Boyer, M. (2001). Gender, Violence, and International Crisis. Journal of Conflict Resolution, 503-518.

Carpenter, C. (2003). 'Women and Children First': Gender, Norms, and Humanitarian Evacuation in the Balkans. International Organization, 661-694.

Churchil, L. B. (2005). Exploring women's complex relationship with political violence: A study of the weathermen, radical feminism and the new left. Florida: University of South Florida Press.

Derisas, F. (29 de Septiembre de 2012). Entre XX X XY...la diferencia es más que una letra. Recuperado el 10 de Marzo de 2014, de Tendencias: http://diario.latercera.com/2012/09/29/01/contenido/tendencias/26-119330-9-entre-xx-y-xy-la-diferencia-es-masque-una-letra.shtml

Diehl, J. (28 de Octubre de 2012). A jihadist group prospers in Syria. Recuperado el 19 de Febrero de 2014, de The Washington Post: http://www.washingtonpost.com/opinions/jackson-diehl-a-jihadist-group-prospers-in-syria/2012/10/28/c036128a-1ed8-11e2ba31-3083ca97c314_story.html

Elgot, J. (26 de Julio de 2013). Syria's Female Rebels: The Islamist Aleppo Brigade Fighting Assad's Forces. Recuperado el 19 de Febrero de 2014, de The Hiffington Post: http://www.huffingtonpost.co.uk/2013/07/26/syria-female-fighters_n_3658205. html

Esman, A. (14 de Febrero de 2014). Who are the women fighting in Syria? Recuperado el 19 de Febrero de 2014, de Breitbar: http:// www.breitbart.com/Big-Peace/2014/02/14/Who-Are-The-Women-Fighting-In-Syria

Gerard, D. (2008). Wanted: Afew Good Women. New York: UNIFEM.

German, L. (2008). Women and the War of Terror. Feminist Review, $140-149$.

Goldstein, J. (2001). War and Gender: How Gender Shapes the War System and Vice Versa. Cambridge: Cambridge University Press.

Jervis, R. (1988). War and Misperception. Journal of Interdisciplinary History, 675-700.

Kolko, G. (1994). Century of War. New York: The New Press.

Lee Ray, J. (2001). Integrating Levels of Analysis in World Politics. Journal of Theoretical Politics, 355-388.

Levy, J. (2008). Counterfactuals and Case Studies. En Oxford Uni- versity, The Oxford Handbook of Political Methodology (págs. 627-644). Oxford: Oxford University Press.

Man, T. S. (1954). Man, The State and War. New York: Columbia University Press.

Mearsheimer, J. (1994). The false promise of international institutions. Cambridge: Harvard University.

OMS. (2014). Género. Recuperado el 2014 de 19 de Febrero, de Organización Mundial de la Salud: http://www.who.int/topics/ gender/es/

Sarkees, M., \& Wayman, F. (2010). Resort to War: 1816 - 2007. CQ Press, 1-32.

Schneider, C., \& Wagemann, C. (2012). Set-Theoretic Methods for the Social Science. A guide to Qualitative Comparative Analysis. New York: Cambridge University Press.

Searcy, S. (1982). The incidence of female criminilaty in the contemporary world by Freda Adler. Contemporary Sociology, 675.

Sjoberg, L. (2010). Women fighters and the "beautiful soul" narrative. International Review of the Red Cross, 53-68.

Syrian Report. (20 de Abril de 2013). Lionesses of National Defence. Recuperado de Syria Report: http://www.syriareport.net/lionesses-of-national-defence/

Varela, N. (2005). Feminismo para principiantes. Barcelona: Ediciones $\mathrm{B}$.

Waltz, K. (1979). Theory of International Politics. New York: Random House.

Zalles, J. (2004). Dominio, sumisión y dependencia. En Z. J, Barreras al diálogo y al consenso (págs. 54-77; 165-172). Quito: Norma.

Zwerman, G. (1997). Participation in undergound organizations: Conservative and feminism images of women associated with armed, clandestine organizations in the United States. London: JAI Press. 